\title{
Intermittent breakdown of current-oscillation tori in n-type GaAs epitaxial layers
}

\author{
U. Margull, J. Spangler, and W. Prettl \\ Institut für Angewandte Physik, Universität Regensburg, 93040 Regensburg, Germany
}

(Received 3 May 1993; revised manuscript received 26 April 1994)

\begin{abstract}
Self-sustained current oscillations in high-purity $n$-type GaAs epitaxial layers were investigated for intermittent behavior in the control parameter plane constituted by the constant bias voltage and an external magnetic field. Intermittent windows were observed in a sequence of Hopf bifurcations. Quasiperiodic and frequency-locked oscillations were found to follow an intermittency type-II or type-III route to chaos in the Pomeau-Manneville classification scheme. Additionally, intermittent bursts due to noise were observed at voltages well below the threshold of bifurcation.
\end{abstract}

\section{INTRODUCTION}

In the past nonlinear dynamical processes in highpurity semiconductors have been investigated in great detail. Impact ionization of residual shallow impurities causes highly nonlinear current-voltage characteristics at temperatures where the thermal energy is smaller than the binding energy of the impurities. At a critical voltage the current rapidly increases transforming the sample into a highly conducting state far from thermal equilibrium. This state is characterized by a filamentary flow of current. ${ }^{1}$ In the course of this nonequilibrium phase transition, self-sustained nonlinear oscillations were observed and different routes to chaos were recognized in various semiconductor materials. ${ }^{2}$

Previous investigations of $n$-type GaAs thin epitaxial layers have shown that the strength and the orientation of an external magnetic field controls the occurrence of complex oscillations and chaotic fluctuations. ${ }^{3}$ Without a magnetic field or with a field parallel to the current flow, at the threshold of breakdown the repetitive ignition and extinction of a current filament yields regular relaxation oscillations. These proceed with increasing average current into a stable filamentary current flow. ${ }^{4}$ Application of a magnetic field in the plane of the epitaxial layer, normal to the current, drives the relaxation oscillations into chaos. This may be due to a nonlinear coupling between the external electric field and the Hall field. ${ }^{5}$ The current filament is not affected in this magnetic field configuration. A magnetic field normal to both the epitaxial layer and the current flow leaves the relaxation oscillations unchanged but destabilizes the filamentary current flow. At magnetic field strengths as small as $10 \mathrm{mT}$ a sequence of two Hopf bifurcations causes quasiperiodic and frequency locked oscillations and, with increasing magnetic field, a third Hopf bifurcation drives the system into chaos following a Ruelle-Takens-Newhouse scenario. ${ }^{6}$ The Hopf bifurcations have been attributed to cyclic generationrecombination processes in the current filament and at the filament borders. ${ }^{7}$ This sequence of oscillatory phenomena is several times repeated in the control parameter plane constituted by the bias voltage and the magnetic field strength.
Reconstructions of the spatial structure of the current filament using a laser scanning microscope ${ }^{7}$ have shown that relatively small magnetic fields increase and decrease the impact ionization probability in the filament boundaries due to accumulation and depletion of mobile charges by the Lorentz force. In the chaotic regime, intermittent burst of the voltage across the sample, associated with Hall voltage collapses, were observed. These indicate that the transition to chaos is accompanied by large lateral rearrangements of the charge distribution. ${ }^{7,8}$

Intermittency is a well known phenomena which has been established in physically very different nonlinear dynamical systems. ${ }^{9-18}$ From the study of simple dissipative systems Pomeau and Manneville derived a classification of intermittency into three types. ${ }^{19}$ While type I and III have been observed in many cases, ${ }^{9,10}$ among them the similar system $p$-type $\mathrm{Ge},{ }^{11-13}$ type II has rarely been established. ${ }^{14-17}$ In ultrapure Ge intermittency of type III has been found close to the threshold of spontaneous formation of traveling space charge domains. ${ }^{18}$

In the present paper, we report on a detailed investigation of intermittent voltage bursts observed in $n$-type GaAs in a parameter regime of filamentary current flow well above the threshold of impact ionization instability. Power spectra, Poincaré sections, the distribution of laminar lengths and the mean laminar length are analyzed in detail as functions of the order parameters. The measurements show that in addition to the previously observed Ruelle-Takens-Newhouse scenario, the $T^{2}$ torus also decays by intermittency. The voltage bursts represent intermittency of types II or III in the PomeauManneville classification scheme. Type-I intermittency may definitely be excluded.

In addition, we present an alternative evaluation technique based on a multichannel scaler which allows very accurate measurements of long time intervals especially for high frequencies in the $\mathrm{MHz}$ range. With this method noise induced intermittency could be analyzed far below the bifurcation point.

\section{EXPERIMENTAL SETUP}

Several epitaxial high-purity $n$-type GaAs layers have been investigated with essentially the same findings. Re- 
sults are reported for the same sample as in Refs. 4 and 8. The layer, grown on a semi-insulating substrate, was of $16 \mu \mathrm{m}$ thickness with alloyed indium Ohmic point contacts $2 \mathrm{~mm}$ apart on the center of opposite edges. The free electron concentration and the mobility at $77 \mathrm{~K}$ were $1.3 \times 10^{14} \mathrm{~cm}^{-3}$ and $8.9 \times 10^{4} \mathrm{~cm}^{2} / \mathrm{V} \mathrm{s}$, respectively, corresponding to a $5.7 \times 10^{14} \mathrm{~cm}^{-3}$ donor density with a compensation ratio of 0.77 . The sample was mounted in the center of a superconducting magnet at $4.2 \mathrm{~K}$. Thermal background radiation was shielded by cold metallic screens. The sample was biased in series with a $100 \mathrm{k} \Omega$ load resistor by a constant voltage source. Time series of the voltage drop across the sample were amplified by a cryogenic junction field effect transistor impedance transformer mounted very close to the sample contacts and recorded by a transient digitizer with 10 bit amplitude resolution. The bandwidth and linear dynamic range of the transformer were $20 \mathrm{MHz}$ and $120 \mathrm{~dB}$, respectively.

For measuring the time distributions of intermittent bursts, a special time interval analyzing method was used, which allowed the analysis of as many as 8192 subsequent time intervals, each ranging from $10 \mathrm{~ns}$ to 80 $\mathrm{ms}$ in duration with a resolution of $5 \mathrm{~ns}$. This is far more than could be achieved by analyzing the time series only and exceeds any previous analysis of intermittency in semiconductors.

In order to achieve such large time ranges, a fast multichannel scaler (MCS 7880 from FAST GmbH, Munich) was used in a rather unusual way. The trigger events were not connected to the counter channels, but to the channel switching input, while a constant $200 \mathrm{MHz}$ signal was fed to the counter. So each channel contained the number of $200 \mathrm{MHz}$ cycles between channel switching events, which was proportional to the time interval. Since each counter channel was 24 bits wide, it was able to count up to $1.6 \times 10^{7}$. This gives, by multiplying by 5 $\mathrm{ns}$, a time range of up to $80 \mathrm{~ms}$ for each interval, with a resolution of 5 ns. Since the MCS 7880 had 8192 channels, we were able to record up to this number of time intervals.

Two different kinds of intermittent events could be detected: voltage bursts, i.e., the sudden growth in the amplitude of a signal, and the collapse of a regular oscillating signal. The voltage signal was first analyzed by a level discriminator of $100 \mathrm{MHz}$ bandwidth, which was able to detect the voltage bursts directly. In the latter case, the discriminator level was chosen so that each oscillation cycle gave a trigger signal. These signals were then fed into a self-built missing pulse detector, that consisted mainly of a retriggerable monostable. Its output remained high as long as it was triggered continuously, but went to low when the triggering signal, i.e., the regular oscillation, disappeared. This low output in turn triggered the MCS 7880 .

\section{RESULTS AND DISCUSSION}

Figure 1 shows a sequence of time series, Poincaré sections and power spectra of self-sustained oscillations measured at $500 \mathrm{mT}$ for three bias voltages $V_{B}$ across the series combination of the sample and the load resistor. At $V_{B}=1.5 \mathrm{~V}$ (top plates) the oscillations contain two frequencies $f_{1}=1.4 \mathrm{MHz}$ and $f_{2}=250 \mathrm{kHz}$. The motion

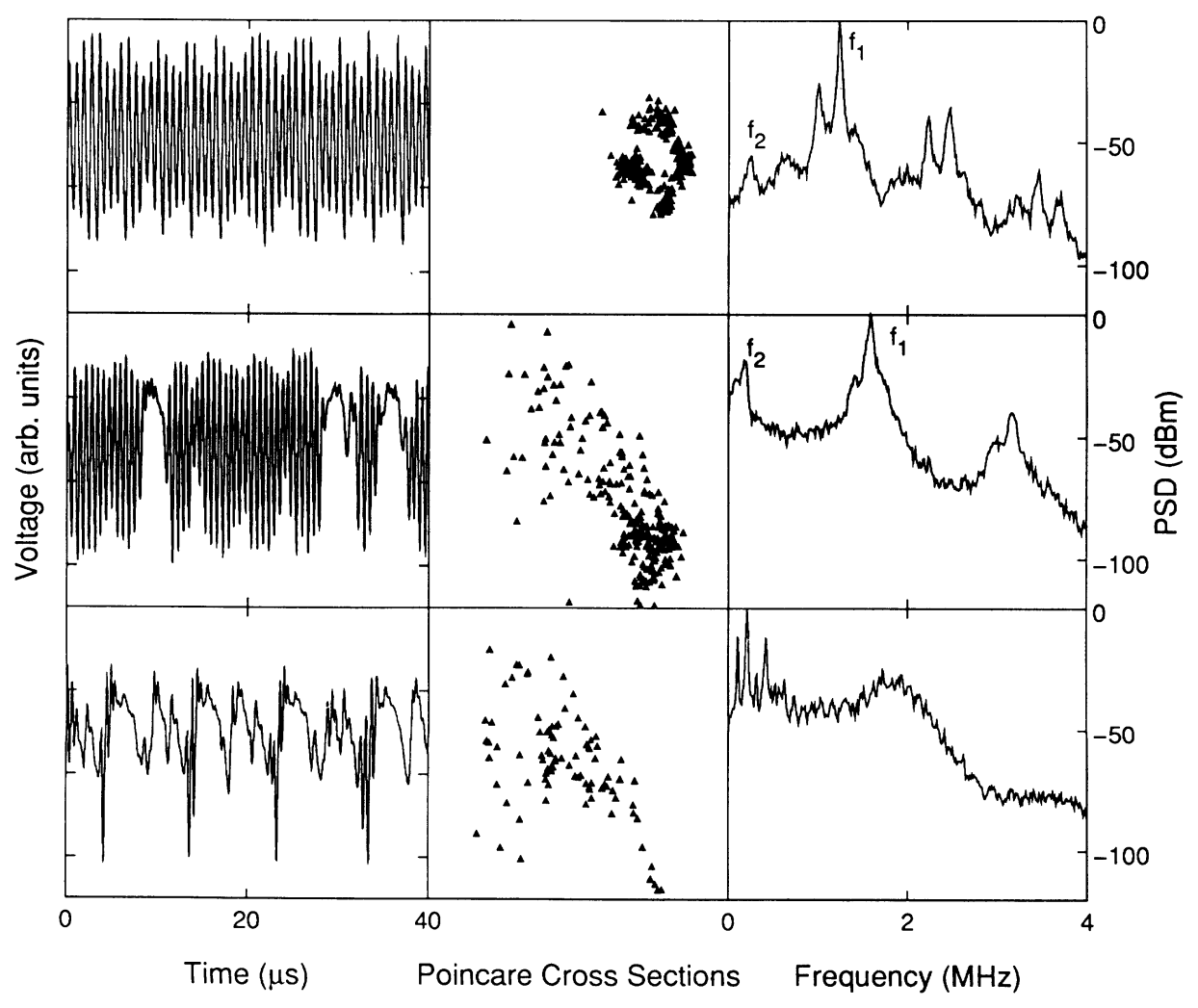

FIG. 1. Time series, Poincaré sections, and power spectra of self-sustained current oscillations for $B=500 \mathrm{mT}$ at three different bias voltages $V_{B}$ : (a) $V_{B}=1.5 \mathrm{~V}$ quasiperiodic motion; (b) $V_{B}=1.8 \mathrm{~V}$ intermittent bursts; (c) $V_{B}=2.0 \mathrm{~V}$ chaotic motion. 
is quasiperiodic on a somewhat noisy torus as shown by the Poincaré section. The distribution of the points on the Poincaré section is not very homogeneous indicating a tendency to mode locking. When the bias voltage is increased (middle plates) intermittent bursts evolve indicating a breakdown of the quasiperiodic oscillations. The bursts are preceded by an increase of the average voltage across the sample up to a critical threshold. As shown previously, these intermittent bursts lead to collapses of the Hall voltage, which does not follow the quasiperiodic oscillations due to the filamentary spatial distribution of the current flow. ${ }^{8}$ Raising the bias voltage further, the laminar phases between two bursts become shorter, until at $V_{B}=2.0 \mathrm{~V}$ the regular oscillation has totally disappeared (bottom plates of Fig. 1). The frequencies $f_{1}$ and $f_{2}$ are no longer observed, the torus has decayed and the system has become chaotic.

In Fig. 2 a different scenario is shown for a smaller magnetic field. Time series, Poincaré sections, and phase portraits are plotted for $B=85 \mathrm{mT}$. At a bias voltage of $1.22 \mathrm{~V}$ (top plates) the oscillations are mode locked with a frequency ratio $1 / 2$. The cycle of period two is clearly to be seen in the phase portrait. Lowering the voltage (middle plates) leads to intermittent bursts in the periodic oscillations with a transition to chaos at $1.18 \mathrm{~V}$ (bottom plates). The oscillations resemble quite close those observed by Richter et al. in $p$-Ge ${ }^{11}$ but we can hardly distinguish an increase of the subharmonic amplitude as a burst is approached. This characteristic precursor of a type-III intermittency has never been observed in the present measurements. For type-I intermittency, the sig- nal should increase monotonically until a burst occurs while type-II intermittency cannot directly be seen from the signal as a function of time. In the present case, there is no obvious change in the signal before or after a burst, with the bursts appearing to occur without warning.

This is further confirmed by analysis of power spectra before and after a burst, which are shown in Fig. 3 . The spectra, which are calculated from the time series in Fig. 1, contain two fundamental frequencies, $f_{1}$ and $f_{2}$, and overtones and mixtures. Before the burst the spectral power at $f_{2} / 2$ increases somewhat as might be expected from type-III intermittency, however, the spectral structure at $f_{2} / 2$ is not very pronounced.

An important signature of intermittency is the distribution $P(t)$ of laminar lengths $t$ and the number of phases $N\left(t_{0}\right)$ lasting longer than $t_{0}$, which for a fixed control parameter $\epsilon$ is given by ${ }^{10}$

$$
N\left(t_{0}\right) \sim \int_{t_{0}}^{+\infty} P(t) d t \sim \sqrt{\frac{e^{-2 \epsilon t_{0}}}{1-e^{-2 \epsilon t_{0}}}}
$$

which in the limit $\epsilon t_{0} \gg 1$ behaves as $N\left(t_{0}\right) \sim e^{-\epsilon t_{0}}$.

In Fig. 4 examples for $P(t)$ and $N\left(t_{0}\right)$ are presented for $B=500 \mathrm{mT}$ (a) below and (b) slightly above the threshold voltage of bifurcation. $P(t)$ shows no temporal upper bound but a long tail at large times. $N\left(t_{0}\right)$ varies like $e^{-\epsilon t_{0}}$ which is characteristic for both type-II and type-III intermittency. ${ }^{20}$ This behavior has been observed at all experimental conditions for which voltage bursts occurred. Thus type-I intermittency may definitely be ruled out because for this a well defined upper

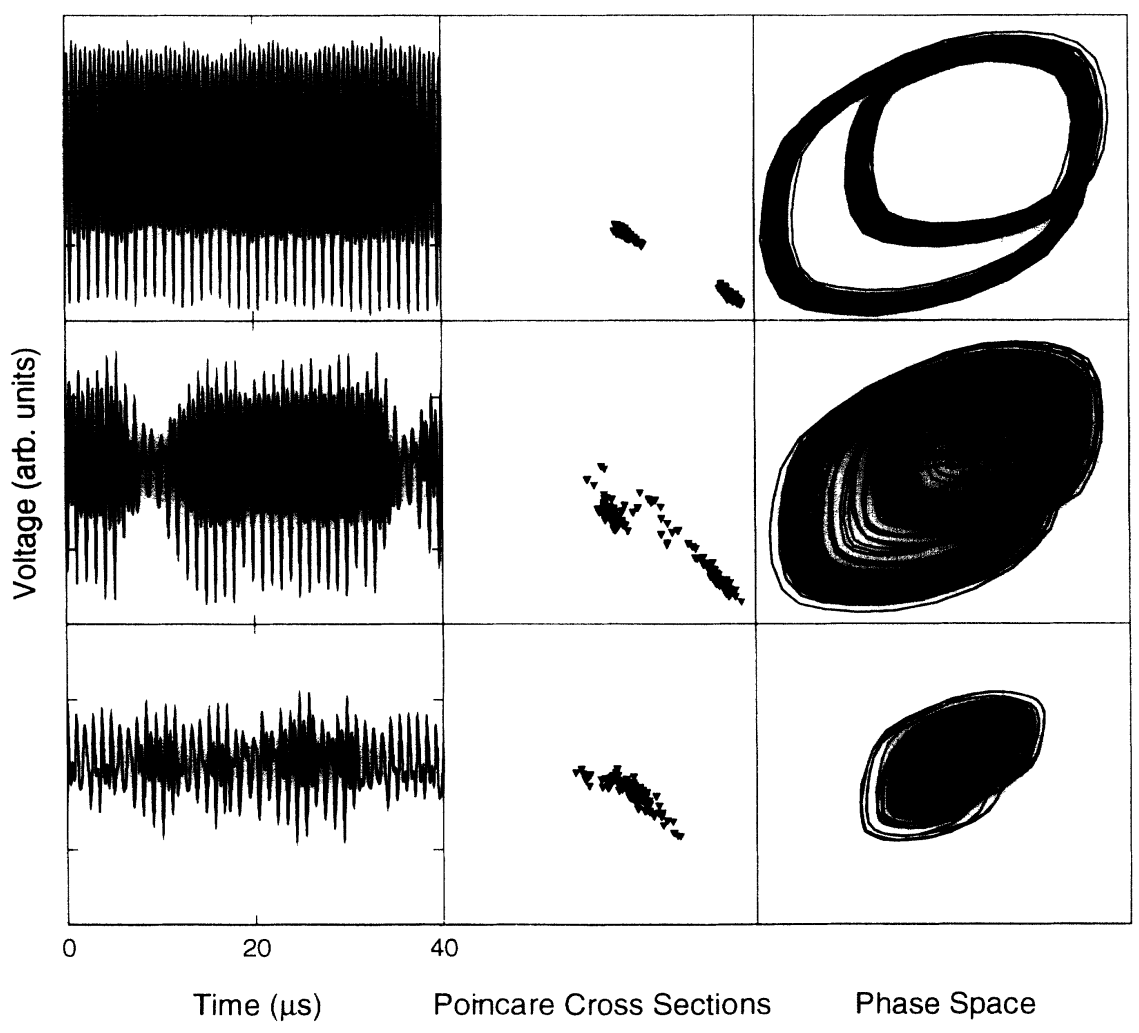

FIG. 2. Time series, Poincaré sections, and phase portrait for $B=85 \mathrm{mT}$ at three different bias voltages $V_{B}$ : (a) $V_{B}=1.22 \mathrm{~V}$ cycle of period two; (b) $V_{B}=1.20 \mathrm{~V}$ intermittent bursts; (c) $V_{B}=1.18 \mathrm{~V}$ chaotic motion. 


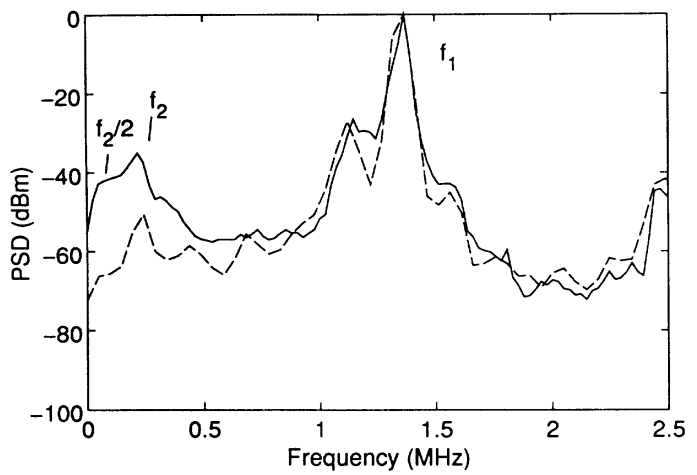

FIG. 3. Power spectra calculated from the time series of Fig. 1 before (solid line) and after a burst (dashed line).

bound of $N\left(t_{0}\right)$ is expected.

Fitting the $N\left(t_{0}\right)$ data to Eq. (1), one gets the control parameter $\epsilon$ as a function of the bias voltage $V_{B}$. The result for $B=500 \mathrm{mT}$ is shown in Fig. 5. In the range from $V_{B}=1.7 \mathrm{~V}$ to $2 \mathrm{~V}$ we have confirmed the presumed linear dependence $\epsilon\left(V_{B}\right)=\left(V_{B}-1.7 \mathrm{~V}\right) / 1.6 \mathrm{~V}$ on the bias voltage $V_{B}$. For voltages smaller than $1.7 \mathrm{~V}$ the underlying mechanism is a different one (see below), so the fitted data should be disregarded.
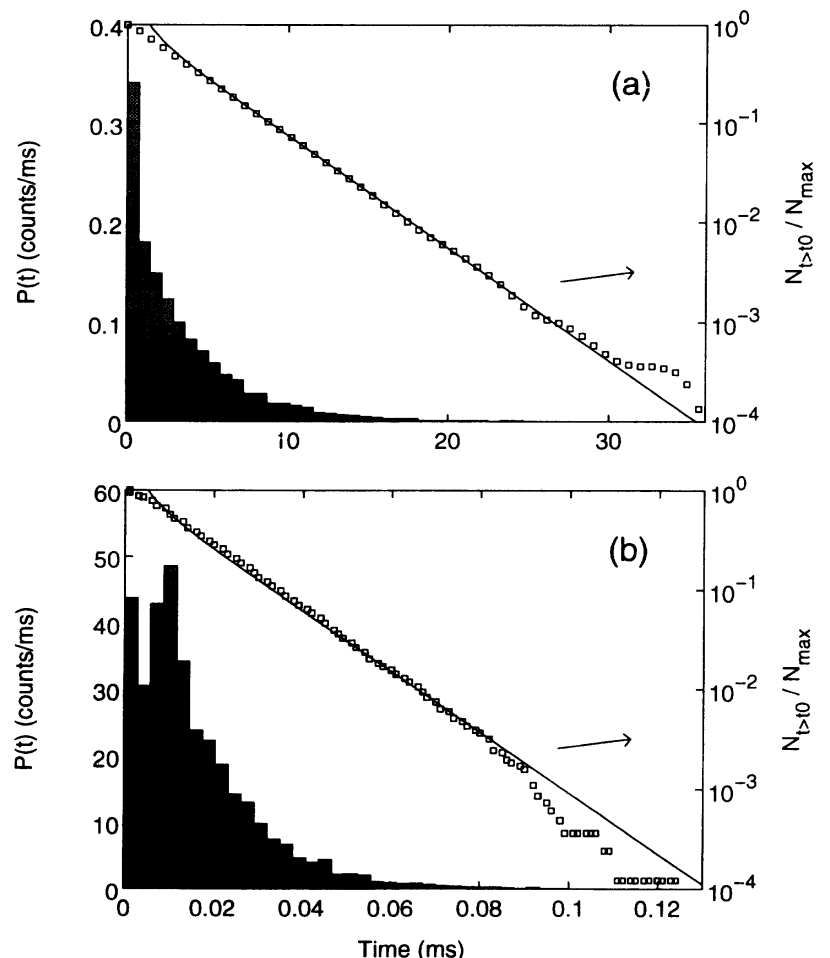

FIG. 4. Distribution $P(t)$ of laminar lengths $t$ (histogram, gray area) and the number of phases $N\left(t_{0}\right)$ lasting longer than $t_{0}$ (small squares) measured at $B=500 \mathrm{mT}$ for two different bias voltages (a) $V_{B}=1.62 \mathrm{~V}$ below and (b) $V_{B}=1.75 \mathrm{~V}$ slightly above the threshold voltage of bifurcation. The solid line shows the expected curve after Eq. (1).

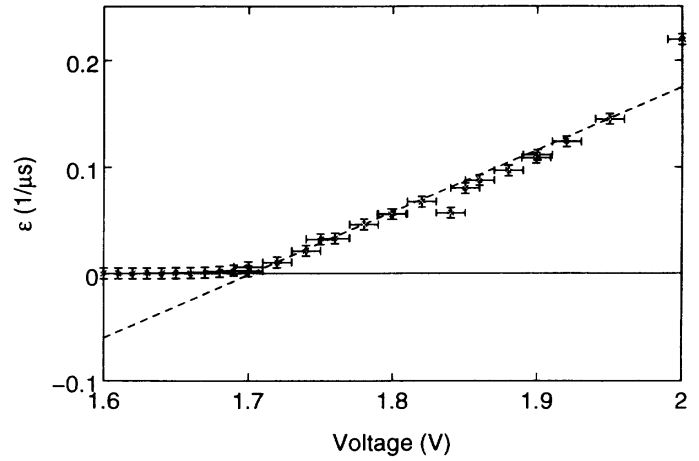

FIG. 5. Theoretical control parameter $\epsilon$, which was calculated from fitting the $N\left(t_{0}\right)$ to $e^{-\epsilon t_{0}}$ as a function of the bias voltage $V_{B}$.

Additional evidence for type-II or type-III intermittency has been found in the dependence of the mean length $\tau\left(V_{B}\right)$ of the laminar phases on the bias voltage $V_{B}$. In Fig. $6 \tau$ and its inverse $1 / \tau$ are plotted versus $V_{B}$ in log-linear and linear-linear representations, respectively. Above a threshold voltage $V_{\text {th }}=1.7 \mathrm{~V}$ the mean laminar length scales as $\tau \sim 1 /\left(V_{B}-V_{\text {th }}\right) \sim 1 / \epsilon$. This result again supports the present interpretation, if one identifies the bias voltage with the control parameter, as shown above, and discriminates from type-I intermittency, in which case we would expect a dependence $\tau \sim 1 / \sqrt{\epsilon}$.

However, for voltages smaller than $V_{\text {th }}$ there are still intermittent bursts, their mean laminar lengths decaying exponentially with rising bias voltage as long as $V_{B}<V_{\mathrm{th}}$. In fact, it is very difficult to determine a threshold voltage by simple inspection of time series since single bursts can be found even far below $V_{\text {th }}$. This indicates a totally different bursting mechanism for voltages $V_{B}<1.7 \mathrm{~V}$. The experimental data do not allow to distinguish between another deterministic process or random noise. Return maps could not be reconstructed because of the constant amplitude of the oscillations between two voltage bursts. Noise, however, seems to be a

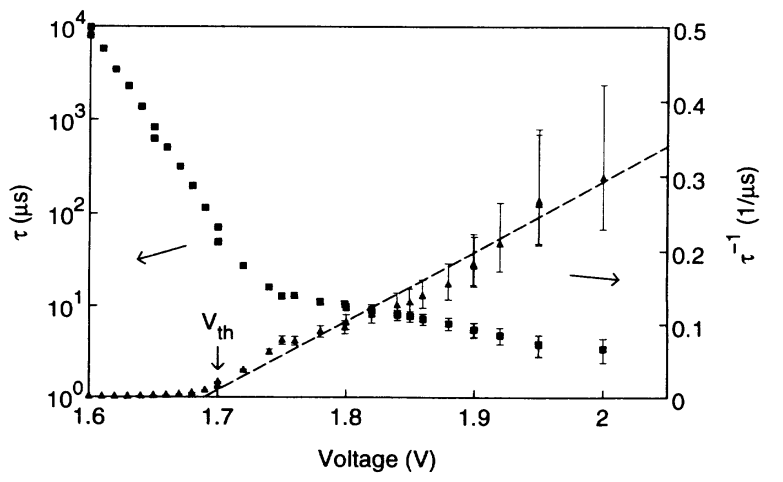

FIG. 6. Mean length $\tau\left(V_{B}\right)$ (a) of the laminar phases and its inverse $1 / \tau$ and (b) as a function of the bias voltage $V_{B}$. 
conceivable mechanism which may have an increased influence on the motion below bifurcation. In the present measurements, the noise to signal ratio varied between about $-50 \mathrm{~dB}$ and $-80 \mathrm{~dB}$ depending on the biasing conditions. This range of noise level is expected from Johnson noise taking into account the drop of the sample resistance with rising average current $(1 \mathrm{M} \Omega$ to $100 \mathrm{k} \Omega$ ) and the fact that the electron temperature is somewhat elevated in the post-breakdown regime. ${ }^{21,22}$ An analogous behavior close to the bifurcation point has been observed in several other intermittent windows with different noise levels. The washing out of the divergence at the bifurcation point is very similar to that found in a theoretical analysis for type-I intermittency. ${ }^{23}$ For voltages $V_{B}>V_{\text {th }}$, we have an unstable fixed point, and the observed bursts reflect the slow journey away from it. For voltages $V_{B}<V_{\mathrm{th}}$, the fixed point is stable, but only in a small region, whose size depends on the bias voltage $V_{B}$. Small random noise could force the system to leave this stable region, which in turn leads to a burst. It is remarkable that even in this voltage range, the distribution of lengths $N\left(t_{0}\right)$ of laminar phases follows an exponential law [Fig. 4(a)]. This is again very similar to the effect of noise on the distribution of laminar lengths observed in Ref. 23 for type-I intermittency.

In addition to the evaluations discussed here in detail, we have determined the fractal dimension $D_{C}$ of the attractor by the method of Grassberger and Proccacia. ${ }^{24}$ The calculations were based on time series for various control parameter sets. In the chaotic regime $D_{C}$ assumed values between 2 and 3 . This shows that higher dimensional spatial intermittency, which is typically observed in turbulent flow, ${ }^{25}$ can be ruled out.

${ }^{1}$ For reviews, see, E. Schöll, Nonequilibrium Phase Transitions in Semiconductors (Springer, Berlin, 1987); Appl. Phys. A 48, 93 (1989), special issue.

${ }^{2}$ K.M. Mayer, R. Gross, J. Parisi, J. Peinke, and R.P. Huebener, Solid State Commun. 63, 55 (1987); A. Brandl, M. Völcker, and W. Prettl, Appl. Phys. Lett. 55, 238 (1989); K. Aoki, T. Kobayashi, and K. Yamamoto, J. Phys. Soc. Jpn. 51, 2373 (1982); S.W. Teitsworth, R.M. Westervelt, and E.E. Haller, Phys. Rev. Lett. 51, 825 (1983); G.A. Held, C. Jeffries, and E.E. Haller, ibid. 52, 1037 (1984).

${ }^{3}$ J. Peinke, A. Mühlbacher, R.P. Huebener, and J. Parisi, Phys. Lett. 108A, 407 (1985); D.G. Seiler, C.L. Littler, R.J. Justice, and P.W. Milomni, ibid. 108A, 462 (1985).

${ }^{4}$ J. Spangler, U. Margull, and W. Prettl, Phys. Rev. B 45, 12137 (1992).

${ }^{5}$ G. Hüpper and E. Schöll, Phys. Rev. Lett. 66, 2372 (1991).

${ }^{6}$ J. Spangler, A. Brandl, and W. Prettl, Appl. Phys. A 48, 143 (1989).

${ }^{7}$ A. Brandl and W. Prettl, Adv. Solid State Phys. 30, 371 (1990).

${ }^{8}$ A. Brandl, W. Kröninger, W. Prettl, and G. Obermair, Phys. Rev. Lett. 64, 212 (1990).

${ }^{9}$ P. Manneville and Y. Pomeau, Phys. Lett. 75A, 1 (1979); P. Bergé, M. Dubois, P. Manneville, and Y. Pomeau, J. Phys. Lett. 41, 341 (1980); Y. Pomeau, J.C. Roux, A. Rossi, S. Bachelart, and C. Vidal, ibid. 42, 271 (1981).

${ }^{10}$ M. Dubois, M.A. Rubio, and P. Bergé, Phys. Rev. Lett. 51, 1446 (1983).

\section{CONCLUSION}

In summary, impact ionization of shallow donors in thin $n$-type GaAs epitaxial films causes regular relaxation oscillations and, for higher average currents, a stable filamentary current flow. A small magnetic field normal to the plane of the sample destabilizes the filament by a sequence of Hopf bifurcations yielding quasiperiodic and mode locked oscillations and finally chaotic fluctuations after a Ruelle-Takens-Newhouse scenario. In the control parameter plane constituted by the magnetic field and the bias voltage, windows occur where the decay of the torus of quasiperiodic and frequency locked oscillations is not due to a Hopf bifurcation but rather by intermittent voltage bursts. It is tempting to attribute this transition to chaos to intermittency of type II, since it is related to an inverse Hopf bifurcation. Our analysis, however, showed that it is not possible to discriminate unambiguously between type-II and type-III intermittency.

Additionally, two different underlying mechanisms for bursting could be found. Above a threshold voltage $V_{\text {th }}$, Pomeau-Manneville intermittency of type II/III and a linear relation between the bias voltage and theoretical control parameter were proven. Below the threshold voltage random noise seems to govern the dynamics of the system, which leads to a different $\tau(\epsilon)$ dependence, but no different distribution $N\left(t_{0}\right)$.

\section{ACKNOWLEDGMENTS}

We thank E. Bauser, Max-Planck-Institut für Festkörperforschung, Stuttgart, for provision of the sample. Financial support by the Deutsche Forschungsgemeinschaft is gratefully acknowledged.

${ }^{11}$ R. Richter, J. Peinke, W. Clauss, U. Rau, and J. Parisi, Europhys. Lett. 14, 1 (1991).

${ }^{12}$ M. Knoop, J. Parisi, W. Clauss, U. Rau, and J. Peinke, Z. Naturforsch. Teil A 46, 1009 (1991).

${ }^{13}$ R. Richter, A. Kittel, G. Heinz, G. Flätgen, J. Peinke, and J. Parisi, Physica D 66, 187 (1993).

${ }^{14}$ P. Richetti, F. Argoul, and A. Arneodo, Phys. Rev. A 34, 726 (1986).

${ }^{15}$ J.-Y. Huang and J.J. Kim, Phys. Rev. A 36, 1495 (1987).

${ }^{16}$ F. Argoul, A. Arneodo, and P. Richetti, J. Phys. (Paris) 49, 776 (1988).

${ }^{17}$ J. Sacher, W. Elsässer, and E. Göbel, Phys. Rev. Lett. 63, 2224 (1989).

${ }^{18}$ A.M. Kahn, D.J. Mar, and R.M. Westervelt, Phys. Rev B 45, 8342 (1992)

${ }^{19}$ Y. Pomeau and P. Manneville, Commun. Math. Phys. 74, $189(1980)$.

${ }^{20}$ P. Bergé, Y. Pomeau, and C. Vidal, Order within Chaos (Hermann, Paris, 1986).

${ }^{21}$ A. Brandl and W. Prettl, Phys. Rev. Lett. 66, 3044 (1991).

${ }^{22}$ V.G. Golubev and W. Prettl, Solid State Commun. 79, 1045 (1991).

${ }^{23}$ J.E. Hirsch, B.A. Huberman, and D.J. Scalapino, Phys. Rev. A 25, 519 (1982).

${ }^{24}$ P. Grassberger and I. Proccacia, Physica D 9, 189 (1983).

${ }^{25}$ H. Daido, Collapse of Tori and Genesis of Chaos in Dissipative Systems (World Scientific, Singapore, 1986). 


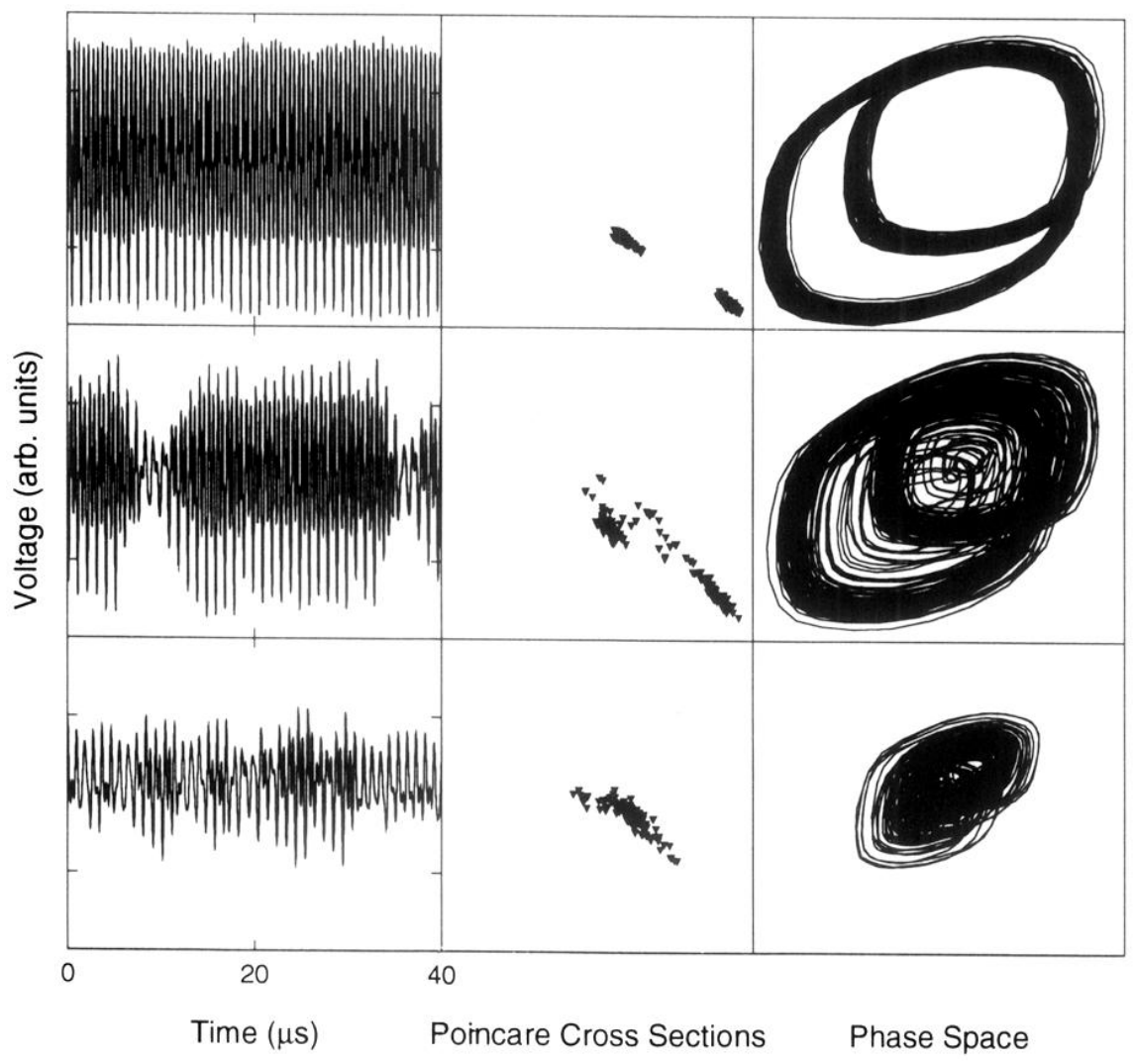

FIG. 2. Time series, Poincaré sections, and phase portrait for $B=85 \mathrm{mT}$ at three different bias voltages $V_{B}$ : (a) $V_{B}=1.22 \mathrm{~V}$ cycle of period two; (b) $V_{B}=1.20 \mathrm{~V}$ intermittent bursts; (c) $V_{B}=1.18 \mathrm{~V}$ chaotic motion. 

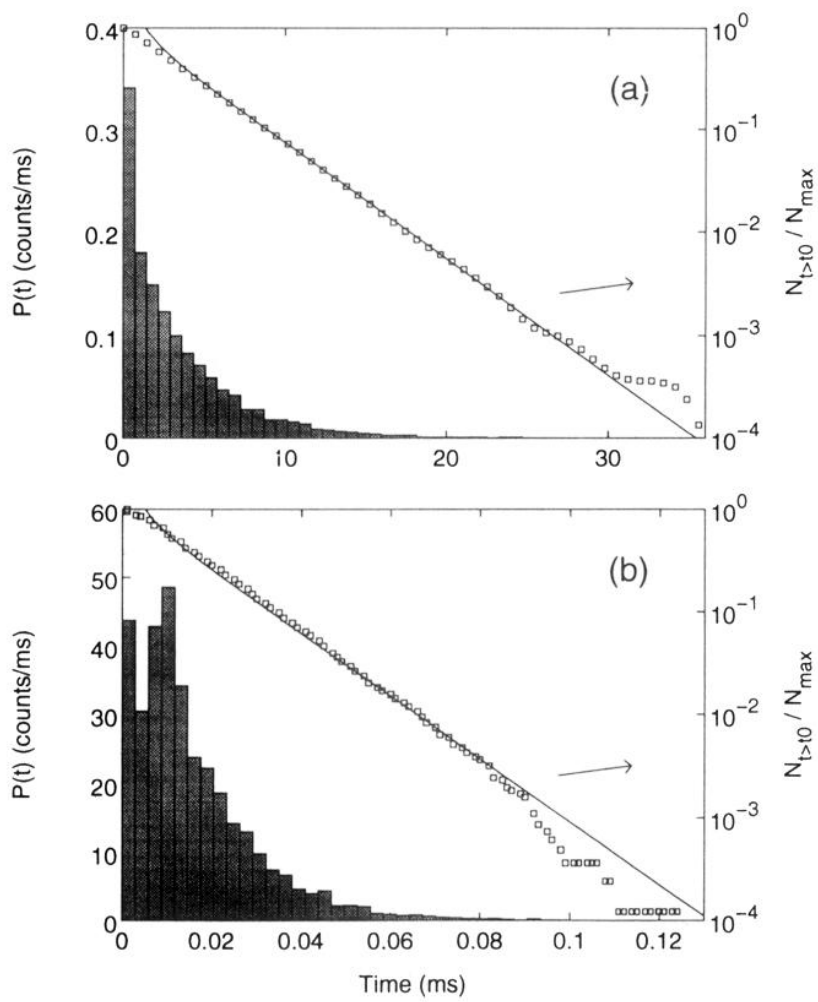

FIG. 4. Distribution $P(t)$ of laminar lengths $t$ (histogram, gray area) and the number of phases $N\left(t_{0}\right)$ lasting longer than $t_{0}$ (small squares) measured at $B=500 \mathrm{mT}$ for two different bias voltages (a) $V_{B}=1.62 \mathrm{~V}$ below and (b) $V_{B}=1.75 \mathrm{~V}$ slightly above the threshold voltage of bifurcation. The solid line shows the expected curve after Eq. (1). 